\title{
Sub-lethal radiation-induced senescence impairs resolution programs and drives cardiovascular inflammation
}

Sudeshna Sadhu' ${ }^{1}$, Christa Decker ${ }^{1}$, Brian E. Sansbury², Michael Marinello ${ }^{1}$, Allison Seyfried $^{4}$, Jennifer Howard ${ }^{4}$, Masayuki Mori ${ }^{3}$, Zeinab Hosseini ${ }^{1}$, Thilaka Arunachalam ${ }^{1}$, Aloke V. Finn ${ }^{3}$, John M. Lamar ${ }^{1}$, David Jourd'heuil ${ }^{1}$, Liang Guo ${ }^{3}$, Katherine C. MacNamara $^{4}$, Matthew Spite ${ }^{2}$, Gabrielle Fredman ${ }^{*}$

${ }^{1}$ The Department of Molecular and Cellular Physiology, Albany Medical College, Albany, NY 12208, USA

${ }^{2}$ Center for Experimental Therapeutics and Reperfusion Injury, Department of Anesthesiology, Perioperative and Pain Medicine, Brigham and Women's Hospital and Harvard Medical School, Boston, MA 02115, USA

${ }^{3}$ CVPath Institute, Gaithersburg, Maryland, USA 2087

${ }^{4}$ The Department of Immunology and Infectious Disease, Albany Medical College, Albany, NY 12208, USA

*Correspondence should be addressed to G.F. (fredmag@amc.edu), The Department of Molecular and Cellular Physiology, ME403, Albany Medical College, Albany, NY 12208, USA. Phone: 518-262-6157, Fax: 518-262-8101

Keywords: radiation, senescence, inflammation-resolution, resolvin, macrophage, efferocytosis, atherosclerosis

Conflict of interests: The authors have declared that no conflicts of interest exist. 


\section{Abstract}

Radiation is associated with tissue damage and increased risk of atherosclerosis but there are currently no treatments and a very limited mechanistic understanding of how radiation impacts tissue repair mechanisms. We uncovered that radiation significantly delayed temporal resolution programs that was associated with decreased efferocytosis in vivo. Resolvin D1 (RvD1), a known pro-resolving ligand, promoted swift resolution and restored efferocytosis in sub-lethally irradiated mice. Irradiated macrophages exhibited several features of senescence, including increased expression of $\mathrm{p} 16^{\mathrm{INK} 4 \mathrm{~A}}$ and $\mathrm{p} 21$, heightened levels of SA- $\beta$-gal, COX-2, and oxidative stress (OS) in vitro, and when transferred to mice exacerbated inflammation in vivo. Mechanistically, heightened OS in senescent macrophages led to impairment in their ability to carry out efficient efferocytosis and treatment with RvD1 reduced OS and improved efferocytosis. Sublethally irradiated $L d l r^{/-}$mice exhibited increased plaque necrosis and $\mathrm{p} 16^{\mathrm{INK} 4 \mathrm{~A}}$ cells compared with non-irradiated controls and treatment with RvD1 significantly reduced these endpoints. Removal of $\mathrm{p} 16^{\text {INK4A }}$ hematopoietic cells during advanced atherosclerosis with $\mathrm{p} 16-3 \mathrm{MR}$ mice reduced plaque necrosis and increased production of key intraplaque resolving mediators. Our results demonstrate that sub-lethal radiation drives macrophage senescence and efferocytosis defects and suggest that RvD1 may be a new therapeutic strategy to limit radiation-induced tissue damage. 


\section{Introduction}

Inflammation-resolution, an active process that tempers pro-inflammatory factors and promotes tissue repair, is controlled by several endogenous mediators that include specialized pro-resolving mediators (SPM) such as lipoxins, resolvins, protectins, and maresins (1). SPMs are protective in vivo, act to control leukocyte trafficking, enhance the clearance of dead cells (i.e. efferocytosis) and promote tissue repair in a manner that does not compromise host defense (1). Dysregulated inflammation-resolution is associated with several prevalent human diseases including atherosclerosis and so understanding processes that may derail these programs are of clinical interest.

lonizing radiation which is broadly used as a treatment for some types of cancers is associated with increased atherosclerosis(2-4). Documented radiation-induced cardiovascular disease (CVD) extends beyond cancer therapy and is also associated with environmental and occupational exposure (5). Radiation is thought to promote atherosclerosis through direct injury to endothelial and smooth muscle cells within the vasculature(2), yet other cellular players are likely involved. Along these lines, macrophages reside in nearly every tissue and, understanding how macrophages respond to radiation is of interest (6). Macrophages are also critical effectors of inflammation-resolution and how radiation impacts temporal resolution programs and efferocytosis are not known.

Radiation can arrest the proliferation of cancer cells but an off target effect is a maladaptive halt in the proliferation of otherwise healthy cells, a process called senescence (7). Radiation-induced senescence provokes the senescence-associated secretory phenotype (SASP) which is associated with increased oxidative stress (OS), 
aberrant metabolic programs, and the release of pro-inflammatory factors (8). There are major gaps in our understanding as to why radiation-induced SASP is left unchecked by our bodies and assessing the mechanisms associated with inflammation and resolution/repair in this context are highly underexplored.

Hematopoietic cells and macrophages are important players in the progression of atherosclerosis $(9,10)$. Sub-lethal radiation leads to hematopoietic stem cell (HSC) senescence and mimics several features of aging and atherosclerosis such as alterations in HSC functions like reduced clonogenicity and skewed differentiation toward myeloid lineages (11). Moreover, human macrophages acquire a pro-inflammatory phenotype when exposed to sub-lethal radiation $(6,12)$. Therefore, we posit that radiation promotes macrophage senescence/SASP and that senescent macrophages contribute to prolonged inflammation and atherosclerosis progression.

Here, we used a model of sub-lethal radiation and observed a significant delay in temporal inflammation-resolution in vivo that was associated with impaired efferocytosis. Treatment with the SPM called Resolvin D1 (RvD1) promoted inflammation-resolution and efferocytosis in the sub-lethally irradiated mice. We found that macrophages do indeed undergo senescence when exposed to radiation and that radiation-induced senescence led to increased OS and defective efferocytosis, that was reversed with the treatment of RvD1 in vitro. Sub-lethally irradiated LdIr/- mice exhibited increased plaque necrosis and p16 $6^{\text {INK4A }}$ cells compared with non-irradiated controls and treatment with RvD1 significantly reduced these endpoints. Conditional removal of hematopoietic p16 ${ }^{\text {INK4 }}$ cells during advanced atherosclerosis reduced plaque necrosis and increased key SPMs in plaques. Together, we found that sub-lethal radiation promotes macrophage 
senescence and that RvD1 can be a new therapeutic strategy to combat radiationinduced damage.

\section{Materials and Methods}

\section{Experimental Animals}

Male 8-10 week old C57BL/6J mice were purchased from Taconic and 8 week old males $L d l r^{\prime-}$ mice were purchased from The Jackson Laboratory. Mice were housed in the Albany Medical College Animal Research Facility. All animal experiments were conducted in accordance with the Albany Medical College IACUC guidelines for animal care and were approved by the Animal Research Facility at Albany Medical College.

\section{$\gamma$-radiation and Zymosan A-induced peritonitis}

Male C57BL/6J mice (Taconic) were mock or sub-lethally $\gamma$-radiated (7 grays) and were given a 3 months recovery period to induce bone marrow myeloid cell senescence (13). After 3 months, mice were intraperitoneally (i.p.) injected with $200 \mu \mathrm{g}$ of ZymA (or ZymA, Sigma, Cat \#Z4250) per mouse. For RvD1 treatment studies, mice were i.p. injected with $300 \mathrm{ng}$ of RvD1 and $200 \mu \mathrm{g}$ of ZymA simultaneously and peritoneal exudates were collected by lavage $24 \mathrm{hrs}$ post injection. For some experiments control or senescent (SC) macrophages $\left(0.8 \times 10^{6}\right.$ cells/mouse) were i.p. injected simultaneously with ZymA and peritoneal cells were collected as described below. Peritoneal cells were harvested at the indicated time points and were then enumerated with a hemocytometer and Trypan blue exclusion. Remaining cells were washed in FACS buffer (PBS containing 5\% (vol/vol) FBS), and labeled with FITC anti-Ly6G (BioLegend cat \#127607) and APC 
anti-F4/80 (eBioscience, cat \#17-4801-82) for 30 minutes at $4^{\circ} \mathrm{C}$. Cells were then washed and resuspended in FACS buffer before performing flow-cytometric assessment. Flowcytometry was carried out on a FACsCalibur (BD Biosciences), and data were analyzed by FlowJo software. Whole bone marrow was flushed from femurs and mRNA was extracted with a Qiagen RNeasy Mini kit (Cat \#74106) and cDNA was synthesized using QuantiTect Reverse Transcription kit (Cat \#205313) according to manufacturer's instructions. Bone marrow mRNA was assessed for $p 16^{I N K 4 A}$, p19ARF and $p 21$ gene expression by qRT-PCR (details below). In parallel, experiments to determine PMN frequency, whole bone marrow was flushed from femurs and tibias and after RBC lysis, cell suspensions were plated and stained using the following antibodies (BioLegend): PECy7-conjugated CD11b (M1/70, cat \#101216), APC-Cy7-conjugated Ly6G (A18, cat \# 127623) and Pacific Blue-conjugated Ly6C (HK1.4, cat \# 128013). Surface stained cells were analyzed on an LSR II (BD Biosciences) with FACSDiva software and analyzed using FlowJo software (TreeStar, Ashland, OR).

\section{Senescent Macrophages (SC-Macrophages)}

Elicited peritoneal macrophages from $\mathrm{C} 57 \mathrm{BL} / 6 \mathrm{~J}$ mice were collected by intraperitoneal (i.p.) injection of ZymA (300 $\mathrm{gg} /$ mouse). Mice were sacrificed $48 \mathrm{hrs}$ post ZymA injection and peritoneal cells were collected by lavage. Cells were enumerated as above and macrophages were plated $\left(700 \times 10^{3}\right.$ cells/well in a 12-well tissue culture plate $)$ in DMEM containing high glucose $(4.5 \mathrm{~g} / \mathrm{L})$ (Corning $10-013-\mathrm{CV}), 10 \% \mathrm{FBS}, 20 \%$ L-cell conditioned media ( $\mathrm{vol} / \mathrm{vol})$ and $1 \%$ Penn Strept overnight. Media was refreshed the next day and the adherent peritoneal macrophages were subjected to 5 grays of $\gamma$-radiation 
and cultured for 3 additional days with the above media. For some experiments, SCmacrophages that had been exposed to $\gamma$-radiation for 2 days were then treated with either Vehicle (PBS) or $10 \mathrm{nM}$ RvD1 for an additional $24 \mathrm{hrs}$.

\section{Quantitative Real-time PCR}

The total RNA was extracted from control or SC-macrophages using a Qiagen RNeasy Mini kit (Cat \#74106) and cDNA was synthesized using QuantiTect Reverse Transcription kit (Cat \#205313) according to manufacturer's instruction. Expression of mRNA was assessed with PerfeCTa SYBR Green FastMix (QuantaBio, VWR, cat\# 101414-288) and run on BioRad CFX Connect real time qRT-PCR machine. Relative expression ( $\Delta \mathrm{Ct})$ was normalized to housekeeping genes, and the $(\Delta \Delta \mathrm{Ct})$ method was used. The sequence for murine primers are as follows: Murine housekeeping gene $18 \mathrm{~S}$ forward: 5'- ATG CGG CGG CGT TAT TCC-3' reverse: 5'-GCT ATC AAT CTG TCA ATC CTG TCC-3', murine p16 ${ }^{\text {INK4A }}$ forward: 5'-AAT CTC CGC GAG GAA AGC-3' reverse: 5'GTC TGC AGC GGA CTC CAT-3', murine p21 forward: 5'-TTG CCA GCA GAA TAA AAG GTG-3', reverse: 5'-TTT GCT CCT GTG CGG AAC-3', murine p19ARF forward: 5'GCC GCA CCG GAA TCCT-3' reverse: 5'-TTG AGC AGA AGA GCT GCT ACGT-3'.

\section{Ki67 staining}

Control or SC-macrophages were prepared as above and plated in an 8-well chambered coverslip (Lab-Tek). Three days post radiation, macrophages were fixed with $100 \%$ Methanol for 5 mins, washed with 1 X PBS and incubated with $0.4 \%$ Titron-X-100 for an additional 10 mins at room temperature. Fixed cells were blocked with $5 \%$ BSA in 
PBS for $1 \mathrm{hr}$ at room temperature and incubated with rabbit anti-mouse Ki67 primary antibody (Abcam ab15580) at 1:200 dilution overnight at $4^{\circ} \mathrm{C}$. The following day macrophages were then washed with 1 X PBS to remove unbound Ki67 antibody and incubated with Alexa Fluor-594 goat anti-rabbit secondary antibody (Invitrogen A-11037) at 1:250 dilution for $2 \mathrm{hrs}$ at room temperature. Nuclei were stained with Hoechst for 10 mins and images were acquired immediately on a Leica SPE confocal microscope and 67 different fields were acquired per well per group. Macrophages whose Ki67 stain colocalized with the nucleus were considered a Ki67 positive cell. The total number of macrophages and Ki67 positive macrophages were counted and expressed as percentage of Ki67 positive cells.

\section{CellROX staining}

Control or SC-macrophages were stimulated with Veh, 10nM RvD1, 10 $\mu \mathrm{M}$ N-AcetylL-cysteine (NAC, ROS inhibitor, Sigma cat\#A7250), or RvD1 and NAC for $24 \mathrm{hrs.} \mathrm{After}$ incubation, cells were stained with $5 \mu \mathrm{M}$ CellROX Green staining solution for 30 mins per manufacturer's instructions (Invitrogen, cat\# C10492). Six-seven different visual fields were acquired with Leica SPE confocal microscope and Mean Fluorescence Intensity (MFI) was calculated using ImageJ.

\section{Metabolic flux analysis}

Control, SC-macrophages or SC-macrophages treated with $10 \mathrm{nM} \mathrm{RvD1}$ as above were seeded (30,000 cells/well) on a Seahorse XF96 cell culture plate (Agilent, \#102601100). The cells were incubated in glucose-free, DMEM (Agilent Cat\# 102353, $\mathrm{pH}=7.4$ at 
$37^{\circ} \mathrm{C}$ ), supplemented with $143 \mathrm{mM} \mathrm{NaCl}$ and $2 \mathrm{mM} \mathrm{L}$-Glutamine for $1 \mathrm{hr}$. After three basal

ECAR measurements, $20 \mathrm{mM}$ Glucose (Sigma, cat \# G8769) followed by $1 \mu \mathrm{M}$ oligomycin, (Sigma, cat\#75351) and then $80 \mathrm{mM}$ 2-deoxy glucose (Sigma, cat\# D6134) were injected in a sequential manner. The ECAR after addition of glucose is reported as fold change.

\section{Senescence-associated beta-galactosidase (SA- $\beta$-gal) assay}

SA- $\beta$-gal activity in control or SC-macrophages was determined using flow cytometry as described in ref (14). Briefly, $700 \times 10^{3}$ macrophages/well were seeded into 12-well plates in complete media, senescence was induced and treated with vehicle or RvD1 as described above. The media was removed, and cells were incubated with 100 $\mathrm{nM}$ bafilomycin $\mathrm{A} 1$ for $1 \mathrm{~h}$ in fresh cell culture medium. C12-FDG $(33 \mu \mathrm{m})$ is a fluorescent $\beta$-gal substrate and was added for additional $1 \mathrm{hr}$ at $37^{\circ} \mathrm{C}$. Macrophages were detached using cell stripper (Corning, cat\#23-25-056-Cl-PK), centrifuged at $250 \mathrm{xg}$ for $5 \mathrm{~min}$ at $4^{\circ} \mathrm{C}$ and resuspended in $0.4 \mathrm{~mL}$ of FACS buffer. Samples were acquired with an LSRII Flow cytometer and analyzed with FlowJo software.

\section{COX-2 staining}

Control and SC-macrophages were fixed with 4\% PFA for 10 mins and permeabilized with 1x perm wash buffer (BD cat\#51-2091KZ) for 30 mins at RT. Fixed cells were stained with rabbit anti-mouse COX-2-Alexa488 at 1:100 dilution in perm wash buffer (Cell signaling, cat \#13596S) for $1 \mathrm{hr}$ at RT. Stained cells were washed with FACS buffer (5\% BSA in PBS) and 10,000 events were collected in BD FACS Calibur. Data was 
analyzed using FlowJo_v10.7.1. Results were expressed as fold change of mean fluorescence intensity of COX-2.

\section{$\mathrm{PGE}_{2}$ ELISA}

Supernatants from control or SC-macrophages were collected and subjected to PGE2 analysis by ELISA (Cayman Chemical).

\section{In vitro Efferocytosis assay}

SC-macrophages were stimulated with Veh, 10nM RvD1 or 10 MM NAC 24 hrs prior to performing efferocytosis. On the day of efferocytosis, Jurkats were enumerated and then stained with PKH26 (Sigma) according to the manufacturer's instructions. Excess dye was removed by washing and the Jurkats were resuspend in RPMI containing 10\% FBS. To induce apoptosis, Jurkats were then exposed to UV irradiation (0.16 Amps, 115 Volts, $254 \mathrm{~nm}$ wavelength) for 15 mins in room temperature and then placed in an incubator $\left(37^{\circ} \mathrm{C}, 5 \% \mathrm{CO}_{2}\right)$ for $3 \mathrm{hrs}(15)$. Apoptotic Jurkats and macrophages were cocultured in a $3: 1$ ratio, respectively for an additional $1 \mathrm{hr}$ in $37^{\circ} \mathrm{C}, 5 \% \mathrm{CO}_{2}$. After $1 \mathrm{hr}$, excess apoptotic cells were removed by washing $\sim 3 x$ with PBS. The cells were then immediately fixed with $4 \%$ formalin and subjected to fluorescence imaging with a Biorad ZOE Fluorescent Cell Imager. Six-to-seven different fields were acquired per well/group and an efferocytic event was considered a macrophage containing red apoptotic cells. Results were expressed as the percent of efferocytosis per total macrophages. 


\section{$\gamma$-radiation Induced Murine Atherosclerosis}

Male $L d l r^{/-}$mice (8 weeks old) were subjected to mock or 7 grays of $\gamma$-radiation and immediately fed a Western Diet (TD.88137, Envigo) for 12 weeks. Mice were socially housed in standard cages at $22^{\circ} \mathrm{C}$ under a $12 \mathrm{hr}$ light and $12 \mathrm{hr}$ dark cycle. During weeks 12-15, WD-fed $L d / r^{\prime-}$ mice were randomly assigned to receive Vehicle (i.e. $500 \mu \mathrm{L}$ of sterile PBS) or RvD1 (100 ng/mouse) for an additional 3 weeks, while still on the WD. Mice were sacrificed at the end of 15 weeks. Lesion and necrotic area analysis were carried out on H\&E-stained lesional cross-sections and were quantified using an Olympus camera and Olympus DP2-BSW software as previously described(16). Briefly, frozen specimens were immersed in OCT, cryosectioned and $8 \mu \mathrm{m}$ sections were placed on glass slides. Atherosclerotic lesion area, defined as the region from the internal elastic lamina to the lumen, was quantified by taking the average of 6 sections spaced $\sim 24 \mu \mathrm{m}$ apart beginning at the base of the aortic root.

\section{Murine lesion p16 $16^{\mathrm{NK} 4 \mathrm{~A}}$ staining}

$\gamma$-radiation induced murine atherosclerosis experiment were performed as described above. Frozen sections were fixed with ice-cold $100 \%$ methanol for 15 mins and washed with $1 \mathrm{X}$ PBS. Fixed sections were incubated with $0.3 \%$ triton 100 for 10 mins and then blocked with blocking buffer (1\% BSA in PBS+0.3\% triton X 100) for $1 \mathrm{hr}$ at room temperature. Sections were incubated with rabbit anti-mouse CDKN2A primary antibody (Abcam ab211542) at 1:100 dilution overnight at $4^{\circ} \mathrm{C}$. The following day sections were then washed with 1 X PBS and incubated with Alexa Fluor-647 goat anti-rabbit secondary antibody (Invitrogen 21246) at 1:500 dilution for 2 hrs at room temperature. Nuclei were 
stained with DAPI for 10 mins and images were acquired immediately on a Leica SPE confocal microscope. Five-six different fields were acquired per mouse section and p16 ${ }^{\text {INK4A }}$ positive cells were counted and expressed as percentage of total lesional cells.

\section{Human plaques}

Human coronary artery specimens with atherosclerotic lesions were selected from individuals enrolled in CVPath Institute Registry with atherosclerosis, a history of being diagnosed with cancer or a history of being diagnosed with cancer and treated with radiation therapy. Briefly, the artery segments were fixed in formalin, and 2 to 3 millimeter segments were embedded in paraffin. Cross-sections of $5 \mu \mathrm{m}$ thick were cut from each of the segments and mounted on slides. Slides were stained with p16 ${ }^{\text {INK4A }}$ (Abcam ab54210) immunohistochemical analyses. Plaque classifications were determined according to our previously published criteria (17). Slides were scanned on Axio.Z1 Slide Scanner (Carl Zeiss, Germany), and image panels were prepared on HALO image analysis platform ver 3.0 (Indica Labs, Corrales, NM).

\section{p16-3MR bone marrow transfers into Ldlr-- mice}

p16-3MR mice were from UNITY Biotechnology, Inc. LdIr/- mice were lethally $\gamma$ irradiated for complete ablation of bone marrow. Radiation were given in two doses, each dose being 4.75 grays with 4 hrs between doses. After the second dose of radiation, we i.v. injected bone marrow cells from the femurs and pelvis of either p16-3MR mice or C57BL6 (WT) control mice into irradiated $L d / r^{\prime-}$ mice. The mice were given antibiotic water (SMZ and TMP, Aurobindo, cat \#NDC 65862-496-47), and a 6 week recovery 
period. After 6 weeks, mice were fed WD for an additional 10 weeks to allow the development of atherosclerosis. At the end of 10 weeks, mice were randomly assigned to receive intraperitoneal injections of either Vehicle (PBS) or $5 \mathrm{mg} / \mathrm{kg} / \mathrm{mouse}$ Ganciclovir (GCV, Sigma cat\#G2536) for the next 3 weeks while still on WD. Mice were sacrificed at the end of 13 weeks and necrotic core analysis was carried out on H\&E-stained lesional cross sections as above.

\section{Identification of Lipid Mediators by Targeted LC-MS/MS}

In order to identify and quantify the lipid mediators in atherosclerotic aortas from Vehicle or GCV treated p16-3MR transplanted $L d l r^{-/}$mice, a targeted liquid chromatography-tandem mass spectrometry (LC-MS/MS)-based analysis was performed as described previously (18). Aortas were isolated and immediately placed in ice-cold methanol. Deuterium-labeled synthetic standards d8-5S-HETE, d5-LXA4, d5-RvD2, d4$\mathrm{LTB}_{4}$ and d4-PGE 2 (Cayman Chemical) were then added to each sample and the tissue was minced on ice. Supernatants were collected after centrifugation $(13,000 \mathrm{rpm}, 10 \mathrm{~min}$, $\left.4{ }^{\circ} \mathrm{C}\right)$ and acidified to $\mathrm{pH}$ 3.5. The samples were then subjected to solid phase extraction using Isolute C18 columns (Biotage). Lipid mediators were eluted from the column following addition of methyl formate and were then concentrated under $\mathrm{N}_{2}$ gas and resuspended in methanol:water (50:50). The samples were analyzed by LC-MS/MS using a Poroshell reverse-phase C18 column $(100 \mathrm{~mm} \times 4.6 \mathrm{~mm} \times 2.7 \mu \mathrm{m}$; Agilent Technologies)-equipped high-performance liquid chromatography (HPLC) system (Shimadzu) coupled to a QTrap 5500 mass spectrometer (AB Sciex) operating in negative 
ionization mode and using scheduled multiple reaction monitoring (MRM) coupled with information-dependent acquisition (IDA) and enhanced product ion-scanning (EPI). Matching the specific retention times and at least 6 diagnostic MS/MS fragmentation ions to those of the synthetic standards run in parallel, lipid mediators were identified in the experimental samples. Using standard curves generated with synthetic standards and accounting for the recovery of the internal deuterium-labeled standards, the abundance of each mediator was then determined and normalized to total protein content.

\section{IMR90 Fibroblast Senescence}

Human fetal lung IMR-90 fibroblast cells were purchased from Coriell Institute for Medical Research, NJ (190-83). Proliferating IMR-90 cells (passage 6-15) were cultured in Eagle's Minimum Essential media (Corning 10-009-CV) with 5\% FBS and 1\% Penn Strept. until $\sim 80 \%$ confluency. IMR-90 cells were detached with trypsin-EDTA (Sigma cat\# 03690), enumerated and plated in a $10 \mathrm{~cm}$ cell culture dish $\left(750 \times 10^{3}\right.$ cells/dish). Cells were cultured overnight ( $16 \mathrm{hrs})$ to allow for attachment and were then subjected to 10 grays of $\gamma$-radiation, and cultured $\left(37^{\circ} \mathrm{C}, 5 \% \mathrm{CO}_{2}\right)$ for 10 days. Fresh media was replaced on day 2, 5 and 8 . On day 10, senescent IMR-90 cells were treated with either Vehicle (PBS, Corning 21-040-CV) or 10nM Resolvin D1 (RvD1) (Cayman Chemical) in serum-free media for additional $24 \mathrm{hrs}$ and end point analyses (all of which are described in detail below) were performed on day 11 . 


\section{Senescence-associated beta-galactosidase (SA- $\beta-g a l)$ Bright field imaging}

SA- $\beta$-gal activity was detected in IMR-90 cells using a SA- $\beta$-gal staining kit (Cell Signaling, \#9860). IMR-90 cells (40,000 cells/well in a 12-well plate) were seeded in complete media, senescence was induced and treated with vehicle or RvD1 as described above. The cells were fixed and incubated for $12-14 \mathrm{hrs}$ at $37^{\circ} \mathrm{C}$ in the presence of the $\beta$ galactosidase staining. Next, cells were imaged on a Zeiss brightfield microscope and 1015 different fields were acquired per well/group. SA- $\beta-$ gal ${ }^{+}$cells were enumerated based on blue staining.

\section{Cholesterol assay}

WD-diet fed Ldl/ ${ }^{/-}$mice were sacrificed and blood was collected in 10\% EDTA by retro-orbital bleeding procedure. Immediately after collection, blood was centrifuged at full speed for 30 mins at $4^{\circ} \mathrm{C}$ and cholesterol assay (Wako, cat\# 999-02601) was performed according to manufacturer's instruction.

\section{Statistical analysis}

For all in vivo studies, mice were randomly assigned to their respective groups. Results are represented as mean \pm S.E.M. Prism (GraphPad Inc., La Jolla, CA) software was used for statistical analysis and statistical differences were determined using the twotailed Student's t-test, One-way ANOVA or Two-way ANOVA with Tukey's multiple comparison post-hoc analysis. Details regarding statistical tests can be found in the figure legends. 


\section{Results}

\section{Sub-lethal radiation delays temporal inflammation-resolution}

To test the impact of radiation on resolution, we first mock or sub-lethally irradiated (IR) male C57/BL6 mice with 7 grays of ionizing radiation. The mice were given a 3 months recovery period to induce hematopoietic cell senescence $(11,13)$. Senescence is mediated by changes in the expression of cell cycle regulators like $p 16^{I N K 4 A}, p 19^{A R F}$ or p21 as examples. Consistent with the literature, we observed that bone marrow from IR mice had significantly higher expression of the senescence markers $p 16^{\text {INK } 4 A}$ (Supplemental Fig. 1A), p19ARF (Supplemental Fig. 1B), p21 (Supplemental Fig. 1C) compared with controls. Accordingly, we also observed other features of senescence and aging including a significant increase in long-term hematopoietic stem cells (LT-HSCs), a decrease in short-term HSC, a strong trend toward increased MMP2s (i.e. erythroid progenitors) (Supplemental Fig. 1D) and a significant decrease in lymphoid-biased MMP4s (Supplemental Fig. 1E). To directly test the role of sub-lethal radiation-induced senescence on the resolution response, we used the widely known in vivo model of Zymosan A (ZymA) induced sterile, self-limited inflammation (19). Control or IR mice were injected with $200 \mu \mathrm{g}$ of ZymA per mouse and peritoneal exudates were collected by lavage 4, 24 and 48 hrs post injection. Leukocytes were enumerated and polymorphonuclear cell (PMN) were assessed by flow cytometry. The peak PMN response $(4 \mathrm{hrs})$ is a measure of inflammation and the time from the peak to when the PMN reach half maximal (e.g. $~ 24 \mathrm{hrs)}$ ) is a quantitative measure of resolution called the resolution interval or $\mathrm{R}_{\mathrm{i}}(19)$. We found that the $\mathrm{R}_{\mathrm{i}}$ for the mock (control) mice was $23 \mathrm{hrs}$ whereas the $R_{i}$ for the IR mice was 35 hrs (Fig. 1A), which suggests an overall delay in 
resolution by $12 \mathrm{hrs}$ in the IR mice. We observed that the PMN numbers were significantly higher in the IR group at $24 \mathrm{hrs}$ (Fig. 1B). Since the timely loss of PMN in tissues is associated with efficient resolution, we next quantified the percent retention of PMN from 4 to 24 hrs in the IR versus control mice. The IR mice had significantly higher PMN from 4 to 24 hrs (Fig. 1C), again suggesting an overall delay in tissue resolution in mice that received a sub-lethal dose of radiation. The modestly reduced influx of neutrophils to the peritoneum at early time points was not due to a loss of bone marrow neutrophils (Fig. Supplemental 1F). Indeed, bone marrow neutrophils in control mice had a significantly higher frequency 24 hrs post ZymA, followed by a significant decline by $48 \mathrm{hrs}$, whereas bone marrow neutrophil frequency in the IR mice did not change throughout the time course (Supplemental Fig. 1F). To rule out non-specific effects of IR, we performed a bone marrow transfer (BMT) in which mice were given a lethal dose of $\gamma$-radiation followed by replenishment of otherwise healthy bone marrow cells from C57/BL6 mice. Lethal doses of $\gamma$-radiation also deplete peritoneal macrophages which are replenished by bone marrow precursors cells upon BMT(20). We found no significant differences in the resolution interval between BMT and non-irradiated control mice (Supplemental Fig. 1G). These results suggest that BMT and thus a replenishment of healthy bone marrow did not delay resolution.

We next questioned whether administration of a key pro-resolving ligand, RvD1 to IR mice would improve resolution endpoints. Indeed, intraperitoneal injection of RvD1 (300 ng/mouse) given with ZymA, significantly decreased PMN numbers at 24 hrs post ZymA (Fig. 1D). Mechanistically, the loss of PMN from the peritoneum during the resolution phase is in part through efferocytosis (21). To determine efferocytosis in vivo, 
we assessed free PMN versus those associated with macrophages using flow cytometry. We observed a significant increase in the free:associated PMN ratio in the IR mice, compared with controls (Fig. 1E), which suggests a defect in efferocytosis. Together, these results provide evidence that sublethal radiation impairs temporal inflammationresolution and deranges efferocytosis in vivo.

\section{Sub-lethal $\gamma$-radiation promotes macrophage senescence/SASP and Resolvin D1}

\section{limits SASP}

Certain peritoneal and elicited macrophages have a proliferative capacity and so we next questioned whether sublethal radiation maladaptively halted their proliferation to promote senescence/SASP. For these experiments, we harvested Zymosan-elicited peritoneal macrophages $48 \mathrm{hrs}$ after ZymA injection. These macrophages were plated, then irradiated (5 grays) and cultured in the presence of L-cell conditioned media for an additional 3 days. Both control and irradiated macrophages excluded Trypan blue equally (not shown). Senescent cells are known to acquire a flattened shape in vitro and irradiated macrophages exhibited an enlarged and flattened shape compared with control macrophages (Fig. 2A). Importantly, irradiated macrophages also exhibited several features of cellular senescence including a significant reduction in proliferation as determined by KI67 staining (Fig. 2B), and a significant increase in p16 ${ }^{\text {INK4A }}$ (Fig. 2C) and p21 expression (Fig. 2D). The expression of p19ARF was not increased in irradiated macrophages compared with non-irradiated controls (Fig. 2E). SA- $\beta$-gal activity is another known marker of senescence, and we used a quantitative flow cytometric method in which control or irradiated macrophages were incubated for an hour with 5- 
dodecanoylaminofluorescein di- $\beta$-D-galactopyranoside (C12-FDG), a fluorescent substrate for $\beta$-galactosidase enzyme (14). Using this approach, we observed that the irradiated macrophages also had a significant increase in SA- $\beta$-gal activity compared with control macrophages (Fig. 2F). We also observed that irradiated macrophages exhibited other features of senescence and SASP including increased COX-2 levels as determined by intracellular staining and flow cytometry (Fig. 2G). Using metabolic flux analysis, we observed that irradiated macrophages had significantly higher extracellular acidification rates (ECAR) after the addition of glucose, consistent with an increase in aerobic glycolysis capacity (Fig. 2H). A representative ECAR tracing is shown on the left and the quantification after addition of glucose is shown on the right (Fig. 2H). Irradiated macrophages also had enhanced oxidative stress (OS) as determine by CellROX staining (Fig. 2I), increased PGE2 levels as determined by ELISA (Fig. 2J) and elevated IL-1 $\alpha$ expression (not shown). Therefore, these results strongly suggest that irradiated macrophages undergo senescence/SASP.

To determine whether RvD1 could reduce senescence/SASP in these cells, we stimulated the senescent (SC)-macrophages with RvD1 (10 nM) 2 days post-radiation and cultured them for an additional $24 \mathrm{hrs}$. Treatment with RvD1 moderately increased the percentage of $\mathrm{Ki}^{+} 7^{+}$macrophages (Fig. 2B) and slightly decreased $p 16^{\mathrm{INK} 4 \mathrm{~A}}$ expression that did not reach significance (Fig. 2C). Also, RvD1 did not modulate the expression of p21 (Fig. 2D) or p19ARF (Fig. 2E), which suggests that RvD1 did not significantly modulate the cell cycle and therefore did not reverse senescence once the macrophage was already committed to this program. We next questioned whether RvD1 modulated the phenotype of the senescent cells. Indeed, RvD1 significantly decreased 
SA- $\beta$-gal activity (Fig. 2F), COX-2 levels (Fig. 2G), aerobic glycolysis (Fig. 2H), OS (Fig. 2I) and PGE2 (Fig. 2J). Indeed, RvD1 significantly reduced OS as potently as the ROS inhibitor, N-acetylcysteine (NAC) (Fig. 2I). The co-treatment of NAC and RvD1 did not further limit OS. Both NAC and RvD1 decreased PGE 2 (Fig. 2J), which suggest that OS may play a role in elevated $\mathrm{PGE}_{2}$ levels in SC-macrophages. Together, these results suggest RvD1 limit several phenotypic features of senescence in macrophages.

\section{Senescent macrophages are poor efferocytes and adoptive transfer of senescent macrophages prolongs inflammation in vivo}

A key function of macrophages is their ability to clear dead cells, a process called efferocytosis. As shown above, SC-macrophages have elevated levels of intracellular ROS. Because previous studies have shown that OS limits efferocytosis, (22) we next questioned whether SC-macrophages have impaired efferocytosis. Indeed, we observed that SC-macrophages have significantly less efferocytosis compared with healthy proliferating controls (Fig. 3A, B). Representative images of efferocytosis are shown in Fig. 3A. Importantly, RvD1 or NAC rescued defective efferocytosis (Fig. 3A, B), which suggests that elevated OS in SC-macrophages limits efficient efferocytosis. These results also suggest that RvD1 rescues efferocytosis through limiting OS in SC-macrophages.

We next questioned whether SC-macrophages exhibited a pro-inflammatory and anti-resolution phenotype in vivo. For these experiments we adoptively transferred either control or SC-macrophages simultaneously with ZymA. Exudates were collected after 4 hrs and PMN were enumerated. We observed that transfer of SC-macrophages had significantly higher PMN numbers at 4 hrs post ZymA injection compared with controls 
(Fig. 3 C). These results suggest that SC-macrophages drive prolonged inflammation in vivo.

\section{RvD1 limits SASP in senescent IMR-90 fibroblasts}

IMR90 fibroblasts are a very well accepted model to study senescence in vitro. Therefore, we also wanted to determine whether this commonly accepted senescence paradigm exhibited similar results as the SC-macrophages. We subjected IMR-90 cells to 10 grays of radiation and then monitored SASP and senescence markers 10 days after radiation (7). First, we found that similar to irradiated SC-macrophages, senescent IMR90 fibroblasts also had a significant decrease in efferocytosis compared with controls (Supplemental Fig. 2A). Consistent with our macrophage results, we observed that senescent IMR90 cells had significantly higher SA- $\beta$-gal activity, which is almost completely abrogated by RvD1 (Supplemental Fig. 2B). Representative images of SA$\beta$-gal staining (blue color) in IMR-90 cells are shown (Supplemental Fig. 2B) and reveal that senescent cells have increased blue staining compared with controls and that RvD1 significantly limited the blue staining (Supplemental Fig. 2B). Additionally, RvD1 dramatically limited $\mathrm{PGE}_{2}$ levels almost to that of control cells (Supplemental Fig. 2C), and significantly decreased aerobic glycolysis based on ECAR (Supplemental Fig. 2D) and ATP resulting from glycolysis (Supplemental Fig. 2E), which again suggests that RvD1 limits key components of the SASP. Lastly, we observed that similar to irradiated SC-macrophages, RvD1 statically significantly, but moderately reduced the expression of the senescence genes, $p 16^{I N K 4 A}$ (Supplemental Fig. 2F), p19ARF (Supplemental Fig. 2G) and p21 (Supplemental Fig. 2H). Together, these data suggest that RvD1 limits 
several phenotypic features of senescent IMR-90 cells which is consistent with the macrophage data.

\section{RvD1 limits necrosis and senescent cell accumulation in sub-lethally irradiated \\ LdIr/- atherosclerotic mice}

Impaired resolution programs and defective efferocytosis are associated with atherosclerosis progression. A consequence of radiation therapy in humans is an increased risk for atherosclerosis (23) and murine models also reveal that radiation exacerbates atherosclerosis $(24,25)$. In agreement with the literature $(24,25)$, we also found that sub-lethal radiation significantly increased plaque necrosis in $\mathrm{Ldll}^{/}$- mice, compared with mock controls (Fig. 4A). Therefore, we next questioned whether treatment with RvD1 could limit plaque necrosis and promote resolution in the context of sub-lethal radiation. For these studies, $L d l r^{\prime-}$ mice were mock or sub-lethally irradiated (IR) and then immediately placed on a Western Diet (WD) for 12 weeks. After 12 weeks on WD, mice were randomly assigned to receive Vehicle or RvD1 (100 ng/mouse) 3x/week for an additional 3 weeks while still on the WD. Mice were then sacrificed, and aortic root lesions were interrogated for necrosis. First, we observed that sub-lethal radiation increased the percent of lesional necrosis compared with mock controls (Fig. 4A). Treatment with RvD1 significantly decreased the percent of lesional necrosis per total lesion area compared with IR mice (Fig. 4A). Body weight (33.75 \pm 0.90 grams for IR, $31.45 \pm 0.87$ grams for IR+RvD1), and plasma cholesterol levels $(1009 \pm 121.3 \mathrm{mg} / \mathrm{dL}$ for IR vs $897.1 \pm 119.5$ $\mathrm{mg} / \mathrm{dL}$ for IR+RvD1) were not different between treatment groups. 
We next questioned if sublethal radiation increased intraplaque $\mathrm{p} 16^{\mathrm{INK} 4 \mathrm{~A}}$ cells. We performed immunofluorescence staining on murine plaques and found that there were significantly more $\mathrm{p} 16^{\mathrm{INK} 4 \mathrm{~A}}$ cells in the IR plaques compared with controls (Fig. 4B, C). Moreover, RvD1 treatment reduced the percentage of lesional p16 ${ }^{\text {INK4A }}$ cells (Fig. 4B, C). Representative immunofluorescence images of lesional p16 $16^{\text {INK } 4 \mathrm{~A}}$ cells are shown in the

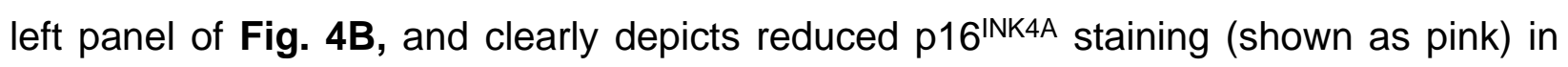
mice treated with RvD1. These results together with our in vitro findings in Fig. 2 suggest that RvD1 may limit initiation of new p16 ${ }^{\mathrm{INK} 4 \mathrm{~A}}$ cells or promote their removal in tissues. Together, these results suggest that RvD1 mitigates sub-lethal radiation-induced atherosclerosis.

Next, we assessed whether radiation leads to increased $\mathrm{p} 16^{\mathrm{INK} 4 \mathrm{~A}}$ cells in human plaques. We obtained human coronary artery specimens with atherosclerotic lesions from individuals enrolled in the CVPath Institute Registry and assessed plaques from patients with atherosclerosis and no history of cancer (NC), plaques from patients who had cancer, but no radiation and (NR), plaques from patients who had cancer and radiation treatment (R). These human plaque sections were stained with $\mathrm{p} 16^{\mathrm{INK} 4 \mathrm{~A}}$ and representative lesional p16 INK4A images for the three groups are shown in Fig. 4D (left panel). First, and in agreement with the literature, we found Immunohistochemical analysis revealed that atherosclerotic plaques exhibited p16 ${ }^{\text {INK4A }}$ cells (Fig. 4D, E), which is consistent with the literature (26) and suggests that atherosclerosis and aging promotes senescence. Importantly, patients who had received radiation therapy had significantly more p16 INK4A cells in the plaques (Fig. 4D, E). Therefore, these results suggest that radiation promotes the accumulation of senescent cells in human plaques. Collectively, these results suggest 
that senescent cells accumulate in advanced plaques and are elevated in the context of sub-lethal radiation.

\section{Conditional removal of p16+ hematopoietic cells limits necrosis and promotes key SPM in advanced atherosclerotic plaques.}

To determine the impact of hematopoietic cell senescence and SPM synthesis in plaques, we took advantage of the p16 driven 3MR (trimodality reporter) fusion protein, which contains functional domains of a synthetic Renilla luciferase (LUC), monomeric red fluorescent protein (mRFP), and truncated herpes simplex virus 1 (HSV-1) thymidine kinase (HSV-TK) (Fig. 5A) (27). The HSV-TK allows killing of $p 16^{+}$cells by ganciclovir (GCV), a nucleoside analog that has a high affinity for HSV-TK but low affinity for the cellular TK. We performed a bone marrow transfer (BMT) from p16-3MR mice into LdIr/mice and after 6 weeks of recovery, mice were placed on WD for 10 weeks. After 10 weeks, mice were randomly assigned to receive Vehicle or GCV (5mg/kg, 3x/week) i.p. injections for an additional 3 weeks while still on WD. (Fig. 5A) . A representative H\&E images of aortic root cross sections display less necrosis in the GCV-treated plaques as outlined by hashed lines compared with Vehicle controls (Fig. 5B, left panel). We also found that there was a significant decrease in lesion area (vehicle $-48693 \pm 1700 \mu \mathrm{m}^{2}$ and GCV - $25838 \pm 3700 \mu \mathrm{m}^{2}$ ) as well as necrosis:lesion area (vehicle $-0.4102 \pm 0.02$ and GCV - $0.2931 \pm 0.04)$ in GCV-treated mice compared with Vehicle. There was no significant change in body weight (vehicle: $35.8 \pm 0.75$ grams and GCV: $34.39 \pm$ 1.01grams), plasma cholesterol (vehicle: $1187 \pm 110.8 \mathrm{mg} / \mathrm{dL}$ and GCV: $960.8 \pm 77.62$ $\mathrm{mg} / \mathrm{dL}$ ) or blood glucose levels (vehicle: $183.1 \pm 9.80 \mathrm{mg} / \mathrm{dL}$ and GCV: $169.9 \pm 5.81 \mathrm{mg} / \mathrm{dL}$ ) 
between the groups. Importantly, we transplanted C57BL6 marrow in Ldlr/- mice and carried out experiments as depicted in Fig. 5A. Using H\&E staining, we found that GCV treatment did not alter lesion area (Vehicle - $54152 \pm 2229 \mu \mathrm{m}^{2}$ and GCV- $55128.16 \pm$ $3467 \mu \mathrm{m}^{2}$ ) or necrotic area (Vehicle - $19125 \pm 714.2 \mu \mathrm{m}^{2} \mathrm{GCV}-19134 \pm 284.8 \mu \mathrm{m}^{2}$ ). Body weight and plasma cholesterol levels were non-significantly different between the groups (data not shown). This suggests that the GCV treatment did not exert any actions on plaque from mice that were transplanted with C57BL/6J bone marrow. Together, these results suggest that hematopoietic senescent cells drive atheroprogression.

We next investigated SPMs in aortic plaques from Vehicle or GCV treated p163MR transplanted Ldlr/- mice by targeted LC-MS/MS analysis. We found that 17-HDHA, i.e. a biosynthetic pathway biomarker to RvD1 (Fig. 5C) and LXA (Fig. 5D) were significantly increased in the GCV-treated plaques compared with Vehicle. A complete list of identified lipid mediators are shown in Supplemental Table I. These results suggest that removal of $\mathrm{p}^{1} 6^{+}$cells during advanced atherosclerosis promotes key SPMs. Collectively, these results suggest a maladaptive role of senescent hematopoietic cells on inflammation-resolution responses in atherosclerosis progression.

\section{Discussion}

The findings of this study provide a previously unappreciated link between radiation, senescence and inflammation-resolution programs which may provide a new framework to approach treatment strategies in contexts where senescent cells accumulate. 
Our human plaque data suggests atherosclerosis is associated with $\mathrm{p} 16^{\mathrm{INK} 4 \mathrm{~A}}$ cells, which is consistent with the literature (26). Moreover, we also found that radiation further increases $\mathrm{p} 16^{\mathrm{INK} 4 \mathrm{~A}}$ cells in human plaques. A long-term consequence of mediastinal radiation is coronary artery disease. Radiation-induced vascular injury is thought to be a major factor that drives long-term occlusive atherosclerotic disease, but there are likely other aspects as well (28). Mediastinal radiation can impact the bone marrow, which is not surprising given that sterna and vertebrae are major sites of hematopoiesis (29). Therefore, it is also possible that even focal thoracic radiation may impact the bone marrow to progress atherosclerosis. Nevertheless, more detailed human studies on bone marrow changes, radiation and atherosclerosis need to be investigated.

Additionally, atherosclerotic plaques have long been associated with the presence of senescent cells, namely endothelial and smooth muscle cells $(30,31)$. Recent data suggests that plaque macrophages may also become senescent (32) and our data herein strongly suggests that senescent hematopoietic cells and macrophages contribute to the progression of atherosclerosis in part but not limited to a defect in SPM synthesis. Along these lines, macrophages are known to proliferate in progressing atherosclerotic plaques, but their proliferation appears to be restricted to a few cycles $(33,34)$. How senescence is involved in the eventual arrest of proliferation of plaque macrophages remains to be explored, but our results provide evidence that removal of senescent hematopoietic cells during advanced atherosclerosis dampens atheroprogression. These findings, along with others point to a critical role of bone marrow cells and macrophages as a critical effectors of plaque progression $(35,36)$. 
Along these lines, sub-lethal radiation in mice mimics several features of hematopoietic aging (37). Indeed, a major risk factor for atherosclerosis is age and how aging impacts the development and progression of atherosclerosis is a critically underexplored arena (38). A previous study showed that bone marrow transfer from aged mice into young $L d l r^{/-}$mice produced larger plaques than $L d l r^{\prime-}$ mice who received young marrow (39). These findings suggest that the bone marrow from aged mice promotes atherosclerosis and a deeper understanding as to which cell types and cues within the aged bone marrow drives atheroprogression are of interest.

Previous reports suggested that macrophages reveal features of senescence, but context and function remained underdeveloped (32, 40, 41). Macrophages are highly responsive to their local tissue microenvironment and can exhibit, pro-inflammatory, profibrotic or even pro-resolving and pro-regenerative functions. A recent study observed that resident peritoneal macrophages from $\mathrm{p} 16^{\mathrm{INK} 4 \mathrm{~A}}$-activated mice exhibited several features of senescence and had increased uptake of Zymosan particles (41). In the context of progressing atherosclerotic lesions, macrophages also exhibited features of senescence, albeit function was not determined (32). These results as well as the findings we present herein suggest that context is critical and the manner in which a cell is driven toward senescence may drive its ultimate phenotype.

Along these lines, in the context of aging and advanced atherosclerosis, the accumulation of senescent cells and their resulting SASP is maladaptive $(42,43)$. However, senescence can also be protective since this is a program that can limit cancer, promote cutaneous wound healing and facilitate patterning during embryogenesis $(8,44)$. Recent reports suggest that senescent cells in arthritic joints can facilitate healing (45). 
Several questions remain in our understanding as to what drives senescent cells toward a tissue reparative versus tissue destructive phenotype and a deeper understanding of senescent cell markers and animal models are needed.

Lastly, we offer a proof-of-concept that RvD1 limits sub-lethal radiation-induced accumulation of senescent cells in atherosclerosis. Currently, there are limited options to quell the SASP or remove senescent cells from tissues. Senotherapeutics are emerging as intriguing new strategies to limit senescent cells in humans (46). Senolytics, for example, inactivate pro-survival mechanisms of senescent cells to promote apoptosis and subsequent clearance. Because efferocytosis is impaired in aging and atherosclerosis $(32,47)$ an increase in apoptotic cells overtime may limit the benefit of senolytics as these apoptotic cells can undergo secondary necrosis. The work presented herein provides an entirely new strategy to limit the most deleterious aspect of senescent cells, i.e., the SASP and suggests that RvD1 may act as a novel senotherapeutic in the context of age-related pathologies. SPMs in general are not immunosuppressive and act to promote tissue repair and regeneration (1) and may be a promising strategy to limit senescent cells in advanced atherosclerosis.

\section{Authors Contributions}

G.F. and S.S. designed experiments and wrote the manuscript. S.S. analyzed all the in vivo and in vitro experiments. S.S. and M.M. performed the in vivo and in vitro experiments. B.E.S. and M.S. performed LC-MS/MS analysis. S.S and C.D. conducted and analyzed macrophage efferocytosis and Cell ROX imaging. T.A. conducted and analyzed IMR-90 efferocytosis experiment. Z.H. and D.J. performed or analyzed 
experiments related to Seahorse. J.M.L helped designed in vivo experiments and with the

preparation of the manuscript. A.S., J.H., and K.C.M. analyzed the bone marrow neutrophil data. M.M, L.G. and A.V.F designed and analyzed the human atherosclerosis experiments.

\section{Acknowledgements}

General: The authors thank Justin Heinz and Nicholas Rymut for their technical expertise.

\section{Source of Funding}

This work was supported by NIH grants HL141127 (G.F.), HL153019 (G.F.), HL142807 (DJ), and R35GM131842 (K.J.M). HL106173 (M.S.), and GM095467 (M.S.). L.G. and A.V.F. are supported by Leducq Foundation Transatlantic Networks of Excellence Grant (18CVD02) to PlaqOmics Research Network.

\section{References}

1. Serhan, C. N., and B. D. Levy. 2018. Resolvins in inflammation: emergence of the pro-resolving superfamily of mediators. J Clin Invest 128: 2657-2669.

2. Stewart, F. A., S. Hoving, and N. S. Russell. 2010. Vascular damage as an underlying mechanism of cardiac and cerebral toxicity in irradiated cancer patients. Radiat Res 174: 865-869.

3. Mertens, A. C., Q. Liu, J. P. Neglia, K. Wasilewski, W. Leisenring, G. T. Armstrong, L. L. Robison, and Y. Yasui. 2008. Cause-specific late mortality among 5-year survivors of childhood cancer: the Childhood Cancer Survivor Study. J Nat/ Cancer Inst 100: 1368-1379.

4. Darby, S. C., M. Ewertz, P. McGale, A. M. Bennet, U. Blom-Goldman, D. Bronnum, C. Correa, D. Cutter, G. Gagliardi, B. Gigante, M. B. Jensen, A. Nisbet, R. Peto, K. Rahimi, C. Taylor, and P. Hall. 2013. Risk of ischemic heart disease in women after radiotherapy for breast cancer. $N$ Engl J Med 368: 987-998.

5. Baselet, B., C. Rombouts, A. M. Benotmane, S. Baatout, and A. Aerts. 2016. Cardiovascular diseases related to ionizing radiation: The risk of low-dose exposure (Review). Int J Mol Med 38: 1623-1641.

6. Meziani, L., E. Deutsch, and M. Mondini. 2018. Macrophages in radiation injury: a new therapeutic target. Oncoimmunology 7: e1494488. 
7. Rodier, F., J.-P. Coppé, C. K. Patil, W. A. Hoeijmakers, D. P. Muñoz, S. R. Raza, A. Freund, E. Campeau, A. R. Davalos, and J. Campisi. 2009. Persistent DNA damage signalling triggers senescence-associated inflammatory cytokine secretion. Nature cell biology 11: 973-979.

8. Wiley, C. D., and J. Campisi. 2016. From ancient pathways to aging cells-connecting metabolism and cellular senescence. Cell metabolism 23: 1013-1021.

9. Swirski, F. K., and M. Nahrendorf. 2016. Bone Marrow Takes Center Stage in Cardiovascular Disease. Circ Res 119: 701-703.

10. Moore, K. J., and I. Tabas. 2011. Macrophages in the pathogenesis of atherosclerosis. Cell 145: 341-355.

11. Shao, L., W. Feng, H. Li, D. Gardner, Y. Luo, Y. Wang, L. Liu, A. Meng, N. E. Sharpless, and D. Zhou. 2014. Total body irradiation causes long-term mouse BM injury via induction of HSC premature senescence in an Ink4a- and Arf-independent manner. Blood 123: 3105-3115.

12. Teresa Pinto, A., M. Laranjeiro Pinto, A. Patricia Cardoso, C. Monteiro, M. Teixeira Pinto, A. Filipe Maia, P. Castro, R. Figueira, A. Monteiro, M. Marques, M. Mareel, S. G. Dos Santos, R. Seruca, M. Adolfo Barbosa, S. Rocha, and M. Jose Oliveira. 2016. Ionizing radiation modulates human macrophages towards a pro-inflammatory phenotype preserving their pro-invasive and proangiogenic capacities. Sci Rep 6: 18765.

13. Wang, Y., B. A. Schulte, A. C. LaRue, M. Ogawa, and D. Zhou. 2006. Total body irradiation selectively induces murine hematopoietic stem cell senescence. Blood 107: 358-366.

14. Debacq-Chainiaux, F., J. D. Erusalimsky, J. Campisi, and O. Toussaint. 2009. Protocols to detect senescence-associated beta-galactosidase (SA-Bgal) activity, a biomarker of senescent cells in culture and in vivo. Nature protocols 4: 1798.

15. Rymut N, H. J., Sadhu S, Hosseini Z, Riley CO, Marinello M, Maloney J, MacNamara KC, Spite M, Fredman G. 2019. Resolvin D1 promotes efferocytosis in aging by limiting senescent cell-induced MerTK cleavage. FASEB J.

16. Fredman, G., J. Hellmann, J. D. Proto, G. Kuriakose, R. A. Colas, B. Dorweiler, E. S. Connolly, R. Solomon, D. M. Jones, E. J. Heyer, M. Spite, and I. Tabas. 2016. An imbalance between specialized pro-resolving lipid mediators and pro-inflammatory leukotrienes promotes instability of atherosclerotic plaques. Nat Commun 7: 12859.

17. Virmani, R., F. D. Kolodgie, A. P. Burke, A. Farb, and S. M. Schwartz. 2000. Lessons from sudden coronary death: a comprehensive morphological classification scheme for atherosclerotic lesions. Arterioscler Thromb Vasc Biol 20: 1262-1275.

18. Dalli, J., R. A. Colas, M. E. Walker, and C. N. Serhan. 2018. Lipid Mediator Metabolomics Via LCMS/MS Profiling and Analysis. Methods Mol Biol 1730: 59-72.

19. Bannenberg, G. L., N. Chiang, A. Ariel, M. Arita, E. Tjonahen, K. H. Gotlinger, S. Hong, and C. N. Serhan. 2005. Molecular circuits of resolution: formation and actions of resolvins and protectins. J Immunol 174: 4345-4355.

20. Cain, D. W., E. G. O'Koren, M. J. Kan, M. Womble, G. D. Sempowski, K. Hopper, M. D. Gunn, and G. Kelsoe. 2013. Identification of a tissue-specific, C/EBPbeta-dependent pathway of differentiation for murine peritoneal macrophages. J Immunol 191: 4665-4675.

21. Schwab, J. M., N. Chiang, M. Arita, and C. N. Serhan. 2007. Resolvin E1 and protectin D1 activate inflammation-resolution programmes. Nature 447: 869-874.

22. McPhillips, K., W. J. Janssen, M. Ghosh, A. Byrne, S. Gardai, L. Remigio, D. L. Bratton, J. L. Kang, and P. Henson. 2007. TNF-alpha inhibits macrophage clearance of apoptotic cells via cytosolic phospholipase A2 and oxidant-dependent mechanisms. J Immunol 178: 8117-8126.

23. Min, S. S., and A. S. Wierzbicki. 2017. Radiotherapy, chemotherapy and atherosclerosis. Curr Opin Cardiol 32: 441-447. 
24. Stewart, F. A., S. Heeneman, J. Te Poele, J. Kruse, N. S. Russell, M. Gijbels, and M. Daemen. 2006. lonizing radiation accelerates the development of atherosclerotic lesions in ApoE-/- mice and predisposes to an inflammatory plaque phenotype prone to hemorrhage. Am J Pathol 168: 649658.

25. Hoving, S., S. Heeneman, M. J. Gijbels, J. A. te Poele, N. S. Russell, M. J. Daemen, and F. A. Stewart. 2008. Single-dose and fractionated irradiation promote initiation and progression of atherosclerosis and induce an inflammatory plaque phenotype in ApoE-/- mice. International Journal of Radiation Oncology* Biology* Physics 71: 848-857.

26. Holdt, L. M., K. Sass, G. Gabel, H. Bergert, J. Thiery, and D. Teupser. 2011. Expression of Chr9p21 genes CDKN2B (p15(INK4b)), CDKN2A (p16(INK4a), p14(ARF)) and MTAP in human atherosclerotic plaque. Atherosclerosis 214: 264-270.

27. Demaria, M., N. Ohtani, S. A. Youssef, F. Rodier, W. Toussaint, J. R. Mitchell, R.-M. Laberge, J. Vijg, H. Van Steeg, and M. E. Dollé. 2014. An essential role for senescent cells in optimal wound healing through secretion of PDGF-AA. Developmental cell 31: 722-733.

28. Weintraub, N. L., W. K. Jones, and D. Manka. 2010. Understanding radiation-induced vascular disease. J Am Coll Cardiol 55: 1237-1239.

29. Geerman, S., S. Hickson, G. Brasser, M. F. Pascutti, and M. A. Nolte. 2015. Quantitative and Qualitative Analysis of Bone Marrow CD8(+) T Cells from Different Bones Uncovers a Major Contribution of the Bone Marrow in the Vertebrae. Front Immunol 6: 660.

30. Matthews, C., I. Gorenne, S. Scott, N. Figg, P. Kirkpatrick, A. Ritchie, M. Goddard, and M. Bennett. 2006. Vascular smooth muscle cells undergo telomere-based senescence in human atherosclerosis: effects of telomerase and oxidative stress. Circ Res 99: 156-164.

31. Minamino, T., H. Miyauchi, T. Yoshida, Y. Ishida, H. Yoshida, and I. Komuro. 2002. Endothelial cell senescence in human atherosclerosis: role of telomere in endothelial dysfunction. Circulation 105: 1541-1544.

32. Childs, B. G., D. J. Baker, T. Wijshake, C. A. Conover, J. Campisi, and J. M. van Deursen. 2016. Senescent intimal foam cells are deleterious at all stages of atherosclerosis. Science 354: 472477.

33. Robbins, C. S., I. Hilgendorf, G. F. Weber, I. Theurl, Y. Iwamoto, J. L. Figueiredo, R. Gorbatov, G. K. Sukhova, L. M. Gerhardt, D. Smyth, C. C. Zavitz, E. A. Shikatani, M. Parsons, N. van Rooijen, H. Y. Lin, M. Husain, P. Libby, M. Nahrendorf, R. Weissleder, and F. K. Swirski. 2013. Local proliferation dominates lesional macrophage accumulation in atherosclerosis. Nat Med 19: 1166-1172.

34. Williams, J. W., K. Zaitsev, K. W. Kim, S. Ivanov, B. T. Saunders, P. R. Schrank, K. Kim, A. Elvington, S. H. Kim, C. G. Tucker, M. Wohltmann, B. T. Fife, S. Epelman, M. N. Artyomov, K. J. Lavine, B. H. Zinselmeyer, J. H. Choi, and G. J. Randolph. 2020. Limited proliferation capacity of aortic intima resident macrophages requires monocyte recruitment for atherosclerotic plaque progression. Nat Immunol 21: 1194-1204.

35. Yvan-Charvet, L., T. Pagler, E. L. Gautier, S. Avagyan, R. L. Siry, S. Han, C. L. Welch, N. Wang, G. J. Randolph, H. W. Snoeck, and A. R. Tall. 2010. ATP-binding cassette transporters and HDL suppress hematopoietic stem cell proliferation. Science 328: 1689-1693.

36. Poller, W. C., M. Nahrendorf, and F. K. Swirski. 2020. Hematopoiesis and Cardiovascular Disease. Circ Res 126: 1061-1085.

37. Young, K., S. Borikar, R. Bell, L. Kuffler, V. Philip, and J. J. Trowbridge. 2016. Progressive alterations in multipotent hematopoietic progenitors underlie lymphoid cell loss in aging. J Exp Med 213: 2259-2267.

38. Tyrrell, D. J., and D. R. Goldstein. 2021. Ageing and atherosclerosis: vascular intrinsic and extrinsic factors and potential role of IL-6. Nat Rev Cardiol 18: 58-68. 
39. Du, W., C. Wong, Y. Song, H. Shen, D. Mori, N. Rotllan, N. Price, A. D. Dobrian, H. Meng, S. H. Kleinstein, C. Fernandez-Hernando, and D. R. Goldstein. 2016. Age-associated vascular inflammation promotes monocytosis during atherogenesis. Aging Cell 15: 766-777.

40. Hall, B. M., V. Balan, A. S. Gleiberman, E. Strom, P. Krasnov, L. P. Virtuoso, E. Rydkina, S. Vujcic, K. Balan, I. Gitlin, K. Leonova, A. Polinsky, O. B. Chernova, and A. V. Gudkov. 2016. Aging of mice is associated with $\mathrm{p} 16(\mathrm{Ink} 4 \mathrm{a})$ - and beta-galactosidase-positive macrophage accumulation that can be induced in young mice by senescent cells. Aging (Albany NY) 8: 1294-1315.

41. Liu, J. Y., G. P. Souroullas, B. O. Diekman, J. Krishnamurthy, B. M. Hall, J. A. Sorrentino, J. S. Parker, G. A. Sessions, A. V. Gudkov, and N. E. Sharpless. 2019. Cells exhibiting strong p16 (INK4a) promoter activation in vivo display features of senescence. Proc Natl Acad Sci U S A 116: 2603-2611.

42. Campisi, J., and L. Robert. 2014. Cell senescence: role in aging and age-related diseases. Interdiscip Top Gerontol 39: 45-61.

43. Pignolo, R. J., J. F. Passos, S. Khosla, T. Tchkonia, and J. L. Kirkland. 2020. Reducing Senescent Cell Burden in Aging and Disease. Trends Mol Med 26: 630-638.

44. Demaria, M., N. Ohtani, S. A. Youssef, F. Rodier, W. Toussaint, J. R. Mitchell, R. M. Laberge, J. Vijg, H. Van Steeg, M. E. Dolle, J. H. Hoeijmakers, A. de Bruin, E. Hara, and J. Campisi. 2014. An essential role for senescent cells in optimal wound healing through secretion of PDGF-AA. Dev Cell 31: 722-733.

45. Montero-Melendez, T., A. Nagano, C. Chelala, A. Filer, C. D. Buckley, and M. Perretti. 2020. Therapeutic senescence via GPCR activation in synovial fibroblasts facilitates resolution of arthritis. Nat Commun 11: 745.

46. Kirkland, J. L., T. Tchkonia, Y. Zhu, L. J. Niedernhofer, and P. D. Robbins. 2017. The Clinical Potential of Senolytic Drugs. J Am Geriatr Soc 65: 2297-2301.

47. Tabas, I. 2010. Macrophage death and defective inflammation resolution in atherosclerosis. Nat Rev Immunol 10: 36-46. 
Figure and Figure legends

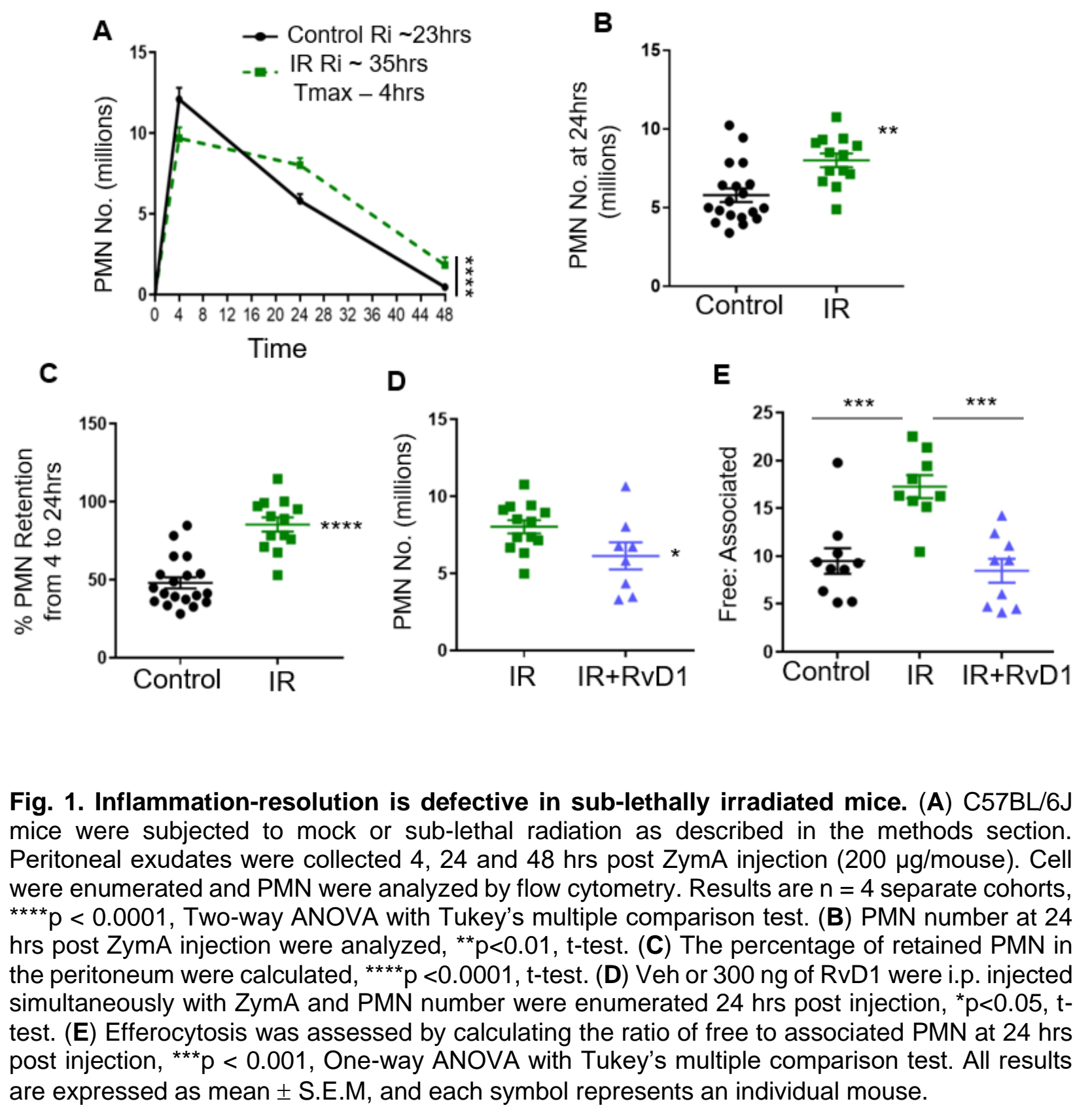



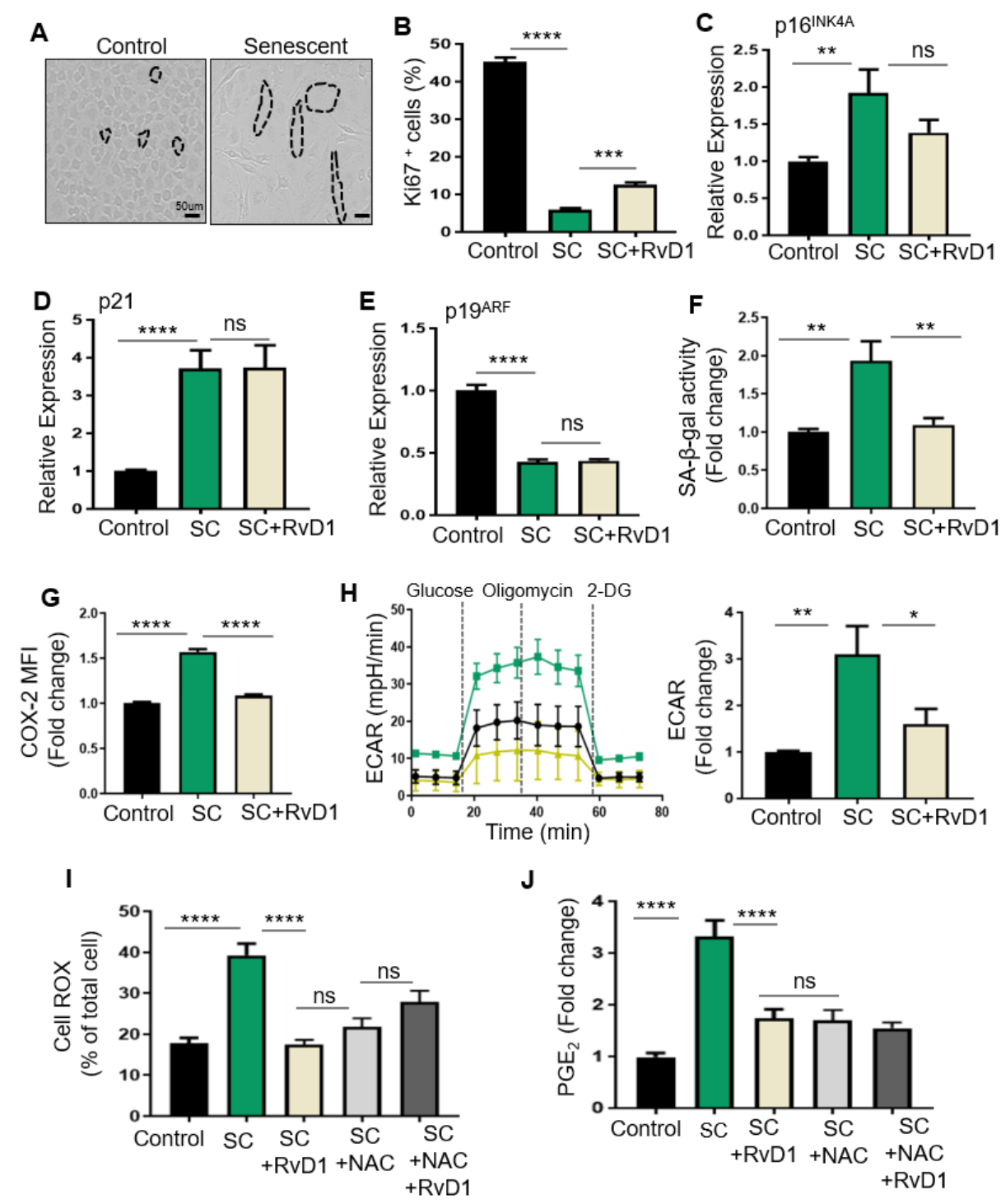

Fig. 2. RvD1 reduces macrophage SASP in vitro. (A) Representative images of control or SCmacrophages. Cells are outlined by blacked hashed lines, Scale bar is $50 \mu \mathrm{m}$. (B) SCmacrophages were treated with vehicle or $10 \mathrm{nM} \mathrm{RvD1}$ for 24hrs, stained with Ki67 and images were acquired on a Lecia confocal microscope and quantified as percentage of Ki67+ cells. (C-E) mRNA expression of p16 ${ }^{\text {INK4A }}$, p21 and p19ARF were measured by qPCR. (F) Control and SCmacrophages were subjected to C12-FDG staining and analyzed by flow cytometry. (G) Control and SC-macrophages were stained with COX-2 and analyzed by flow cytometry. (H) Representative ECAR tracing (left) and ECAR quantification after addition of glucose (right) for control and SC-macrophages were assessed by glycolysis stress test using the Agilent Seahorse XFe96 Analyzer. For $\mathbf{G}$ and $\mathbf{H}$, Macrophages pooled from 4 mice and performed 2 separate times. (I) SC-macrophages were treated with either 10nM RvD1 or $10 \mu \mathrm{M}$ NAC for $24 \mathrm{hrs}$. CellROX Green fluorescence was detected using Leica confocal microscope. Results are expressed as percentage of total cells. (J) Supernatants from control and SC-macrophages were subjected to $\mathrm{PGE}_{2}$ ELISA analysis. Results are expressed as fold change to control. All results are mean \pm S.E.M., analyzed by One-way ANOVA with Tukey's post-hoc test. ${ }^{*} p<0.05,{ }^{* *} p<0.01,{ }^{* * *} p<0.001$, ${ }^{* * * *} \mathrm{p}<0.0001$, ns - non-significant. $\mathrm{n}=3$ independent experiments unless otherwise specified. 
A

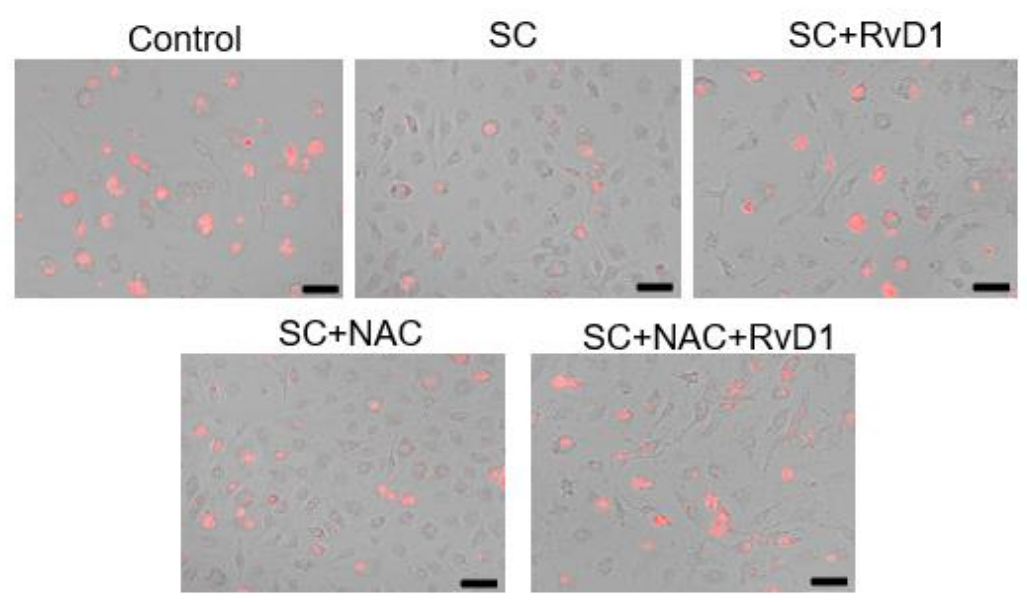

B

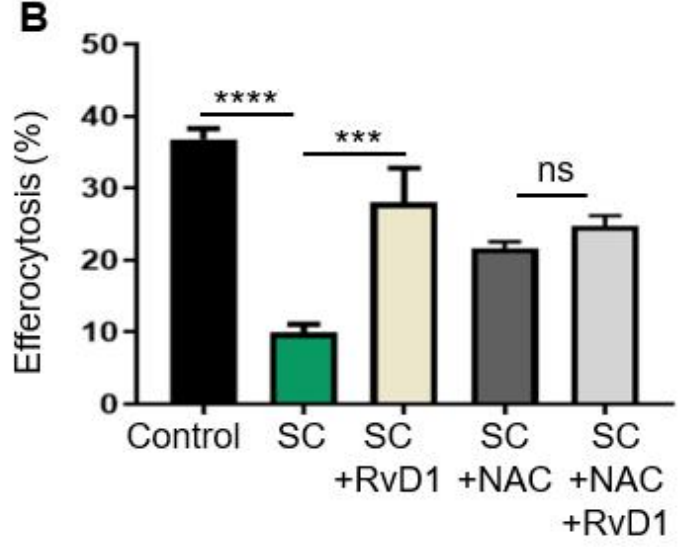

C

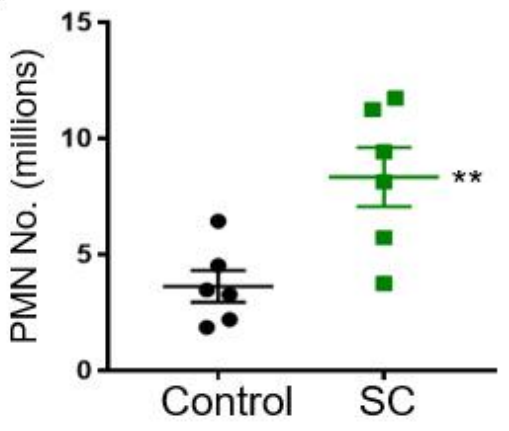

Fig. 3. Senescent macrophages are poor efferocytes. (A, B) Efferocytosis was performed as explained in the methods and representative images $(\mathbf{A})$ and quantification $(\mathbf{B})$ of efferocytosis is shown. Macrophages are displayed with brightfield imaging and apoptotic cells are shown in red. The total number of macrophages with internalized apoptotic cells were counted as an efferocytic event and results are expressed as percentage. Scale bar is $50 \mu m, n=3$ independent experiments performed in quadruplet. ${ }^{* * *} \mathrm{p}<0.001$, ${ }^{\star * \star *} \mathrm{p}<0.0001$, ns-non-significant, One-way ANOVA with Tukey's post-hoc test. (C) Simultaneous i.p. injection of ZymA with control or senescent macrophages was performed. PMN were collected 4 hrs post injection and enumerated by flow cytometry. ${ }^{* *} p<0.01$, t-test. All results are expressed as mean \pm S.E.M. and each symbol represents an individual mouse. 


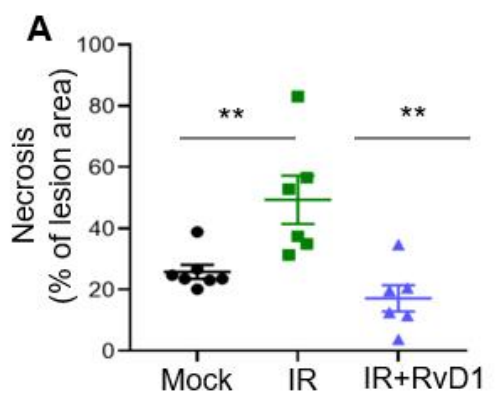

B

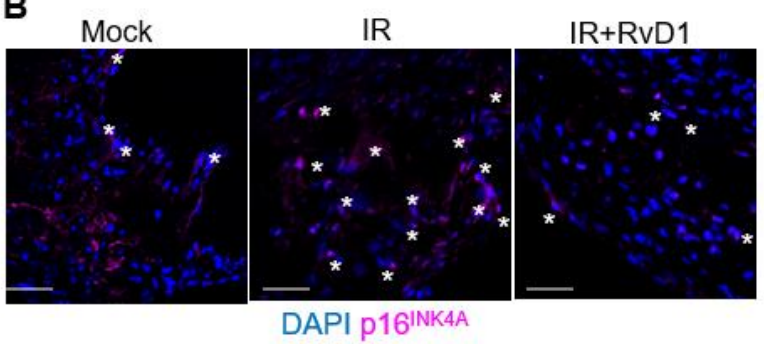

D

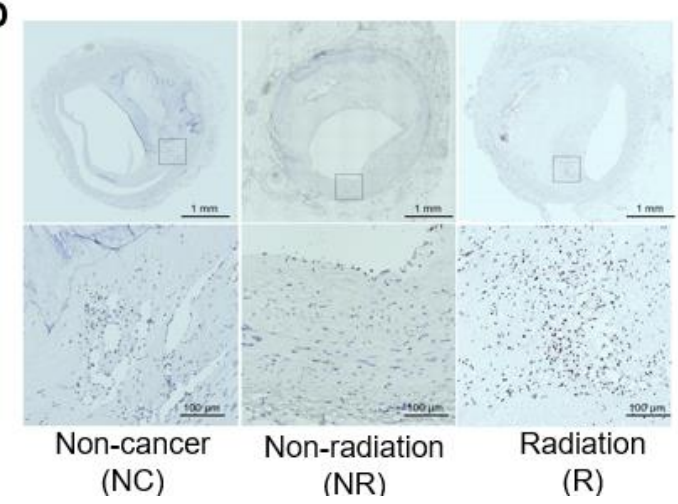

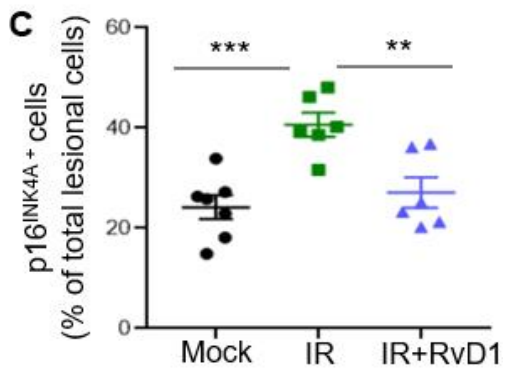

E

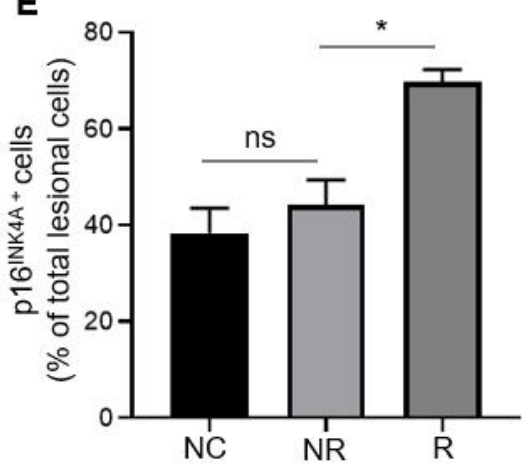

Fig. 4. RvD1 limits necrosis and $\mathrm{p} 16^{\mathrm{INK} 4 \mathrm{~A}+}$ cells in progressing plaques from sub-lethally irradiated $L d \boldsymbol{d} \boldsymbol{r}^{/-}$mice. (A) Quantification of percent necrosis of lesion area) in mock, Vehicle or RvD1 treated sub-lethally radiated- $L d l r^{-/}$mice. (B) Representative $\mathrm{p} 16^{\text {INK4A }}$ immunofluorescence images of Mock (left panel), IR (middle panels) or IR+RvD1 (right panels) plaques. White stars represent $\mathrm{p} 16^{\mathrm{INK} 4 \mathrm{~A}+}$ cells. $\mathrm{p} 16^{\mathrm{INK} 4 \mathrm{~A}}$ is shown in magenta, and nuclei are stained in blue with DAPI. Magnification 40X, Scale bar is $50 \mu \mathrm{m}$. (C) Quantification of p16 INK4A+ lesion cells. (D, E) Immunohistochemistry of $\mathrm{p} 16^{\mathrm{INK} 4 \mathrm{~A}}$ in human coronary atherosclerotic lesions from non-cancer patients (NC), patients diagnosed with cancer but not treated with radiation (NR) and those with cancer and radiation treatment (R). (D) The whole artery cross-sections were shown on the top, a higher magnification (within the boxed region) is shown on the bottom. The scale bars are $1 \mathrm{~mm}$ and $200 \mu \mathrm{m}$, respectively and display p16 ${ }^{\mathrm{INK} 4 \mathrm{~A}+}$ cells in brown. (E) Quantification of the percent of p16 ${ }^{\text {INK4A }}$ cells per total lesional cells is shown from five different arterial beds from 3 separate patients pre group. $(\mathbf{A}, \mathbf{C})$ Each symbol represents an individual mouse. All results are expressed as mean \pm S.E.M., analyzed by One-way ANOVA with Tukey's post-hoc test. ${ }^{*} p<0.05,{ }^{* *} p<0.01$, ${ }^{* * *} \mathrm{p}<0.001$, ns - non-significant. 


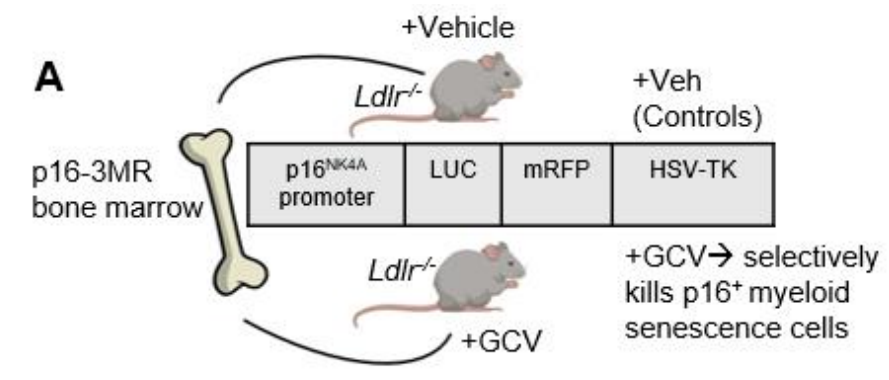

B

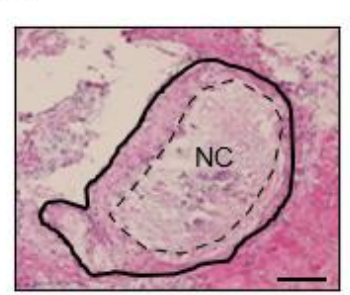

+ Vehicle p16-3MR $\rightarrow$ LdIr $^{-1-}$

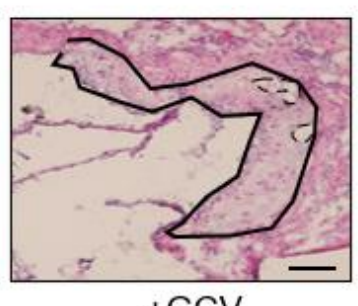

$+\mathrm{GCV}$

p16-3MR $\rightarrow$ LdIr $^{-1}$
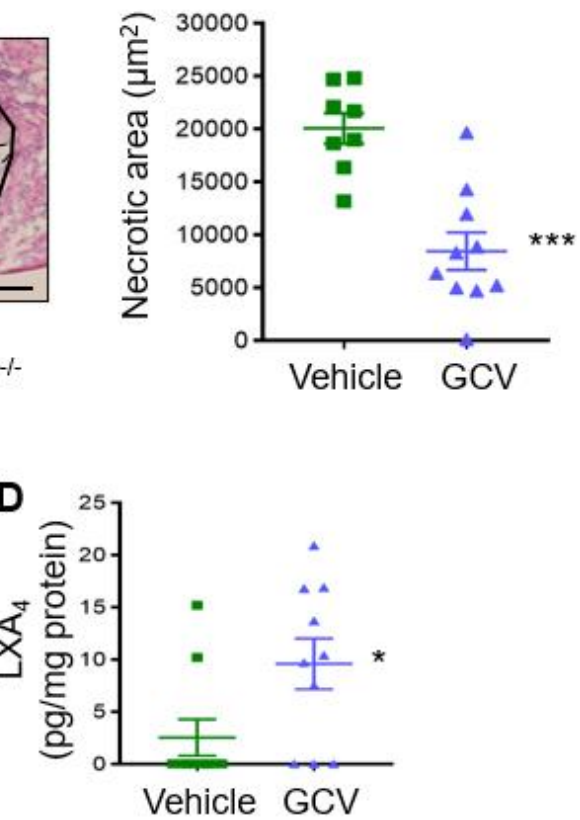

Fig. 5. Removal of $\mathrm{p}^{1} 6^{+}$cells during advanced atherosclerosis limits necrosis and promotes key SPMs. (A) Scheme depicting the p16-3MR bone marrow transfer model. (B) Representative aortic root images of Vehicle or Ganciclovir (GCV, $5 \mathrm{mg} / \mathrm{kg}, 3 x /$ week, i.p.) treated p16-3MR $\rightarrow L d I^{\prime /}$ are shown on the left. Lesions are outlined with black solid lines and necrotic regions are outlined with blacked hashed lines. Quantification of lesional necrosis is shown on the right, ${ }^{* * *} \mathrm{p}<0.001$, t-test. (C, D) $17-\mathrm{HDHA}$ or $\mathrm{LXA}$ were quantified by LC-MS/MS analysis, and lipid mediators are expressed in $\mathrm{pg} / \mathrm{mg}$ of protein, ${ }^{*} \mathrm{p}<0.05, t$-test. For all experiments, each symbol represents an individual mouse and data are shown as mean \pm S.E.M. 